\title{
The Value in the links...
}

- The conceptualisation of GVC links... Definitions

- The structure of GVC links... Trends \& Patterns

- GVC Link formation mechanisms... Drivers

- Shaping economic \& social outcomes... Impacts 


\section{Buyers and Suppliers of A Product...}

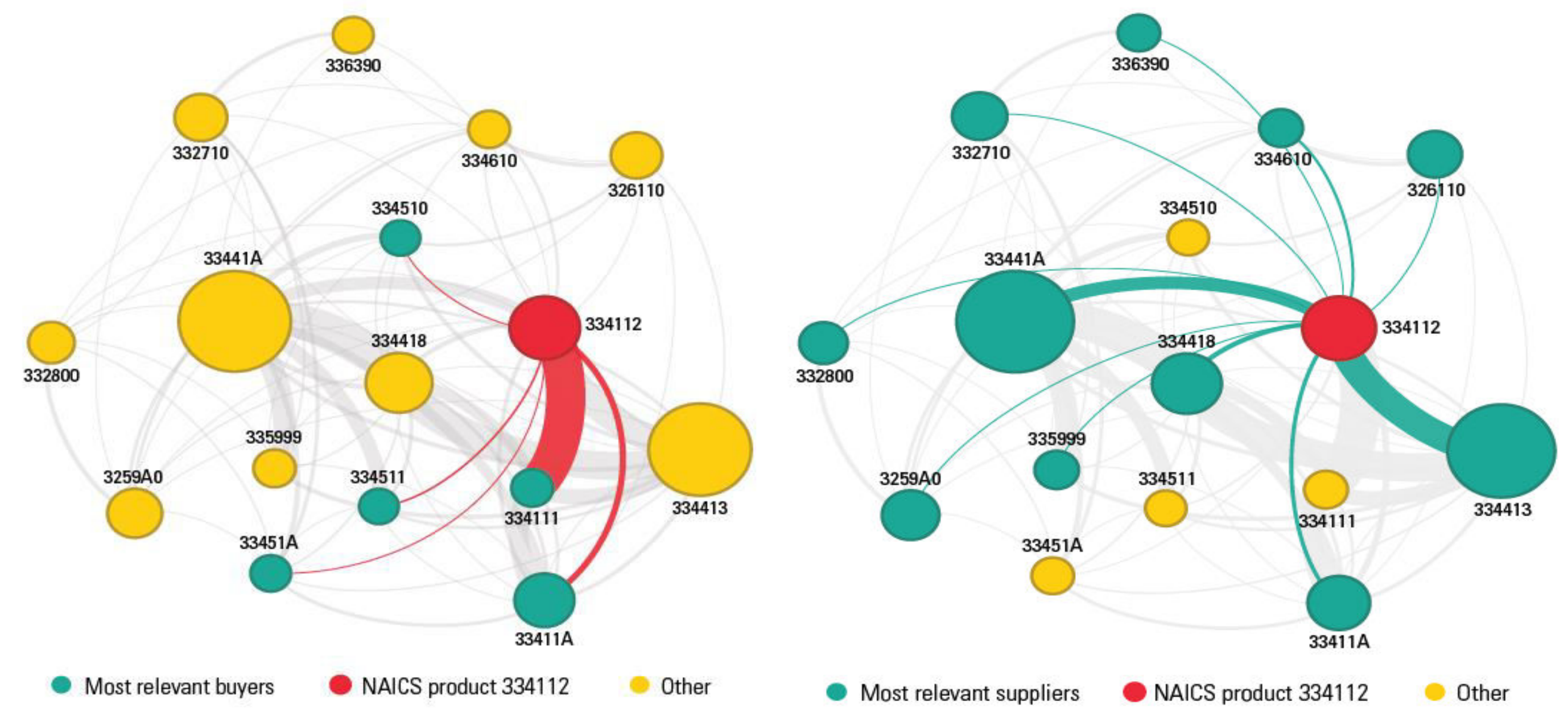

Source: Santoni and Taglioni forthcoming. Adapted from Benchmark Input-Output Data, Bureau of Economic Analysis, U.S. Department of Commerce. 


\section{About Data...}

- Gross export and gross import data of HS 1988/92 6-digit product classification covering 30 years starting from 1988 to 2017

- Manufacturing products requires exclusion of agriculture products and extractive industries.

- So we exclude following HS 2-digit codes: 1-24 (animal, vegetable, and food products), 25-27 (minerals and fuels), 44-48 (a large part of wood), 68-69, and 71 (a large portion of stone and glass) from our downloaded HS 6-digit products.

- Around 2900 to 3500 products for each year in manufacturing gross import and gross export data.

- Data for exports and imports in each year contain HS 6-digit codes, product descriptions, trade flow codes, quantity units, quantities, net weights, trade values. The unit of trade values is 1000 US Dollar. 


\begin{tabular}{|c|c|c|c|}
\hline $\begin{array}{l}\text { VC Stage/ } \\
\text { Subassembly }\end{array}$ & $\begin{array}{l}\text { HS Codes } \\
\text { (2002) }\end{array}$ & HS Code Descriptions & VC Sector \\
\hline $\begin{array}{l}\text { Passenger } \\
\text { vehicles }\end{array}$ & $\begin{array}{l}870321 \\
870322 \\
870323 \\
870324 \\
870331 \\
870332 \\
870333\end{array}$ & $\begin{array}{l}\text { 870321, } 870322,870323,870324 \text { : Other vehicles, with spark- } \\
\text { ignition internal combustion reciprocating piston engine } \\
<1000 \mathrm{cc}, 1000-1500 \mathrm{cc}, 1500-3000 \mathrm{cc} \text {, }>3000 \mathrm{cc} \\
87033 \text { : Other vehicles, with compression-ignition internal } \\
\text { combustion piston engine (diesel or semi-diesel) }<1500 \mathrm{cc} \text {, } \\
1500-2500 \mathrm{cc},>2500 \mathrm{cc}\end{array}$ & - \\
\hline Body system & 870600 & $\begin{array}{l}\text { 8706: Chassis fitted with engines, for the motor vehicles of } \\
\text { headings } 87.01-87.05\end{array}$ & Chassis \\
\hline Drive train & $\begin{array}{l}840733 \\
840734 \\
840820\end{array}$ & $\begin{array}{l}\text { Reciprocating piston engines used for the propulsion of } \\
\text { vehicles of Chapter } 87 ; \text { of a cylinder capacity: } \\
>250 \mathrm{cc} \leq 1,000 \mathrm{cc} \\
>1,000 \mathrm{cc} \\
\text { Compression-ignition internal combustion piston engines } \\
\text { (diesel or semi-diesel engines]; of a kind used for the } \\
\text { propulsion of vehicles of Chapter } 87\end{array}$ & Engine \\
\hline $\begin{array}{l}\text { Body system } \\
\text { (suspension) }\end{array}$ & $\begin{array}{l}401110 \\
401211 \\
870831^{+} \\
870839^{+} \\
870870 \\
870880 \\
870894\end{array}$ & $\begin{array}{l}\text { 401110: New pneumatic tires, of rubber; of a kind used on } \\
\text { motor cars } \\
\text { 401211: Retreaded tires; of a kind used on motor cars } \\
\text { (including station wagons and racing cars) } \\
\text { Brakes and servo-brakes and parts thereof; } \\
\text { 870831: Mounted brake linings } \\
\text { 870839: Other } \\
\text { 870870: Road wheels and parts and accessories thereof } \\
870880 \text { : Suspension systems and parts (incl. shock absorbers) } \\
\text { 870894: Steering wheels, columns and boxes }\end{array}$ & $\begin{array}{l}\text { Tires } \\
\text { Brakesn } \\
\text { Wheels } \\
\text { Suspension systems } \\
\text { and parts (incl. } \\
\text { shock absorbers) } \\
\text { Steering wheel }\end{array}$ \\
\hline $\begin{array}{l}\text { Body system } \\
\text { (panels) }\end{array}$ & $\begin{array}{l}870710 \\
700711 \\
700721 \\
830230\end{array}$ & $\begin{array}{l}\text { 870710: Bodies (incl. cabs), for motor vehicles of headings } \\
\text { 87.01-.05; for the vehicles of heading } 87.03 \\
\text { 700711: Toughened (tempered) safety glass, of size and shape } \\
\text { suitable for use in vehicles, aircraft, spacecraft or vessels } \\
\text { 700721: Laminated safety glass.. } \\
\text { 830230: Other mountings, fittings and similar articles suitable } \\
\text { for motor vehicles }\end{array}$ & $\begin{array}{l}\text { Body Panels } \\
\text { Windows/ } \\
\text { Windshield } \\
\text { Metal mountings }\end{array}$ \\
\hline $\begin{array}{l}\text { Body system } \\
\text { (front \& rear } \\
\text { end modules) }\end{array}$ & $\begin{array}{l}870810 \\
870891 \\
870892 \\
842139 \\
853910\end{array}$ & $\begin{array}{l}\text { Parts and accessories of the motor vehicles of headings 87.01- } \\
\text { 87.05; } \\
\text { 870810: Bumpers and parts thereof } \\
\text { 870891: Radiators } \\
\text { 870092: Silencers and exhaust pipes } \\
\text { 842139: Filtering or purifying machinery and apparatus for } \\
\text { gases; Intake air filters for internal combustion engines; other } \\
\text { 853910: Electric filament or discharge lamps, including sealed } \\
\text { beam lamp units and ultra-violet or infra-red lamps; arc-lamps; } \\
\text { Sealed beam lamp units }\end{array}$ & $\begin{array}{l}\text { Bumpers } \\
\text { Radiators } \\
\text { Silencers } \\
\text { (mufflers)/exhaust } \\
\text { Filters } \\
\text { Headlights }\end{array}$ \\
\hline \multirow{2}{*}{$\begin{array}{l}\text { Body system } \\
\text { (interior) }\end{array}$} & $\begin{array}{l}940120 \\
870821\end{array}$ & $\begin{array}{l}\text { 940120: Seats of a kind used for motor vehicles } \\
\text { 870021: Safety seat belts }\end{array}$ & \begin{tabular}{|l|} 
Seats \\
Seatbelts^
\end{tabular} \\
\hline & $\begin{array}{l}852721^{1} \\
852729^{1}\end{array}$ & $\begin{array}{l}\text { 85272: Radio-broadcast receivers not capable of operating } \\
\text { without an external source of power, of a kind used in motor }\end{array}$ & \begin{tabular}{|l|l|} 
Electronic \\
Instruments:
\end{tabular} \\
\hline
\end{tabular}

\begin{tabular}{|c|c|c|c|}
\hline $\begin{array}{c}\text { VC Stage/ } \\
\text { Subsssembly }\end{array}$ & $\begin{array}{c}\text { H5 Codes } \\
{[2002]}\end{array}$ & HS Code Descriptions & VC Sector \\
\hline & 910400 & $\begin{array}{l}\text { vehicles, including apparatus crpable of receiving also radio- } \\
\text { telephony or radio-telegraphy } \\
\text { 910400: Instrum ent panel docks and clocks of a similar type } \\
\text { for vehicles, aircraft, spacecraft or vessels. }\end{array}$ & $\begin{array}{l}\text { Radios } \\
\text { Clocks }\end{array}$ \\
\hline $\begin{array}{l}\text { Body system } \\
\text { (other) }\end{array}$ & 870029 & $\begin{array}{l}\text { 870029: Parts and accessories of the motor vehicles of } \\
\text { headings 87.01-87.05. Other parts and accessories of bodies } \\
\text { (including cobs); Other }\end{array}$ & Other \\
\hline & $\begin{array}{l}840991 \\
840999\end{array}$ & $\begin{array}{l}\text { 84099: Parts suitable for use solely or principally with the } \\
\text { engines of heading } 104.07-08 \text {. }\end{array}$ & Engine parts \\
\hline Drive train & $\begin{array}{l}870040 \\
870850 \\
870860^{+} \\
870893\end{array}$ & $\begin{array}{l}\text { Parts/accessories of motor vehicles of headings } 87.01-05 \text {; } \\
\text { 870840: Gear boves } \\
\text { 870850: Drive-axles with differential, whether or not prowided } \\
\text { with other trangmission components } \\
\text { 870860: Non-driving axles and parts thereof } \\
\text { 870893: Other parts/accessories; Clutches \& parts thereof }\end{array}$ & $\begin{array}{l}\text { Gear boves: } \\
\text { Drive-anles } \\
\text { Clutches: }\end{array}$ \\
\hline $\begin{array}{l}\text { Body System/ } \\
\text { Drive train }\end{array}$ & 870099 & $\begin{array}{l}\text { 870899: Parts and accessories of the motor vehicles of } \\
\text { headings 87.01-87.05.0ther parts and accessories, Other }\end{array}$ & \begin{tabular}{|l|} 
Other \\
Airbag $^{\mathrm{m}}$ \\
\end{tabular} \\
\hline \multirow{5}{*}{$\begin{array}{l}\text { Electrical } \\
\text { Equipment }\end{array}$} & $8507^{*|c|}$ & $\begin{array}{l}\text { 8507: Electric accumulators, including separators therefor, } \\
\text { whether or not rectangular (including square)" }\end{array}$ & \begin{tabular}{|l|} 
Batteries \& partz \\
(accumulators)
\end{tabular} \\
\hline & $8511^{*|7|}$ & $\begin{array}{l}\text { 8511- Electrical ignition or starting equipment of a kind used } \\
\text { for spark-ignition or compression-ignition internal combustion } \\
\text { engines (for example, ignition magnetos, magneto-dynamos, } \\
\text { ignition coils, sparking plugs and glow plugs, tarter motors]; } \\
\text { generators (for example, dynamos, alternators) and cut-outs of } \\
\text { a kind used in conjunction with such engines. }\end{array}$ & Ignition \& parts \\
\hline & 854430 & $\begin{array}{l}\text { 854430: Ignition wiring sets and other wiring sets of a kind } \\
\text { used in vehicles, aircraft or ships }\end{array}$ & Wire harnesses \\
\hline & $\begin{array}{l}851220 \\
851230 \\
851240 \\
851290\end{array}$ & $\begin{array}{l}\text { 8512- Electrical lighting or signa ling equipment (excl. articles of } \\
\text { heading } 85.39 \text {, windscreen wipers, defrosters and demisters } \\
\text { used for cycles or motor vehicles. NOTE: all of } 8512 \text { except } \\
851210 \text { (pertains to bicycles). }\end{array}$ & $\begin{array}{l}\text { Sgnaling } \\
\text { Lighting/visual, } \\
\text { sound, windscreen } \\
\text { wipers, parts }\end{array}$ \\
\hline & 841520" & $\begin{array}{l}\text { 841520: Air conditioning machines, of a kind used for perzons } s_{r} \\
\text { in motor vehicles }\end{array}$ & Air Conditioners \\
\hline
\end{tabular}




\section{Top Imports \& Exports 2017}

\begin{tabular}{|c|c|}
\hline Rank & ProductCode \\
\hline 1 & 851790 \\
\hline 2 & 852520 \\
\hline 3 & 854140 \\
\hline 4 & 847330 \\
\hline 5 & 847193 \\
\hline 6 & 852810 \\
\hline 7 & 880240 \\
\hline 8 & 854211 \\
\hline 9 & 841112 \\
\hline 10 & 870899 \\
\hline
\end{tabular}

\section{ProductDescription}

Parts of electrical apparatus for I Transmission apparatus, for radiote Photosensitive semiconductor device Parts and accessories of automatic Storage units, whether or not prese Television receivers including vide Aircraft nes of an unladen weight e Monolithic integrated circuits, dig Turbo-jets of a thrust exceeding 25 Motor vehicle parts nes
Import Value in 1000 USD

11284403.94

7943789.405 4540262.67

3157816.507

2801751.042

2704122.257

2395660.368

2251533.249

2022660.643

1861818.66
\% Share 5.652755631 3.979324076 2.274377584 1.581861579 1.403495839 1. 35459014 1.200070709

1. 127872355

1. 013222001

0.932650583

\begin{tabular}{|c|c|}
\hline Export Value in 1000 USD & \% Share \\
\hline 9656236.04 & 5.603555992 \\
\hline 3475237.959 & 2.016695782 \\
\hline 2520017.214 & 1.462377007 \\
\hline 2425630.855 & 1.40760419 \\
\hline 2209565.257 & 1.282220379 \\
\hline 1989325.43 & 1. 154414244 \\
\hline 1962342.354 & 1. 138755848 \\
\hline 1713061.678 & 0.994097181 \\
\hline 1699799.197 & 0.986400906 \\
\hline 1673470.992 & 0.971122533 \\
\hline
\end{tabular}

0.986400906

\section{ProductDescription}

Other medicaments of mixed or unmix Automobiles with reciprocating pist Motor vehicle parts nes Copper cathodes and sections of cat Aluminium unwrought, not alloyed Floating docks and vessels which pe Aircraft parts nes T-shirts, singlets and other vests, Motorcycles with reciprocating pist Cotton, not carded or combed 
Top 100 Manufacturing Imports in 2017
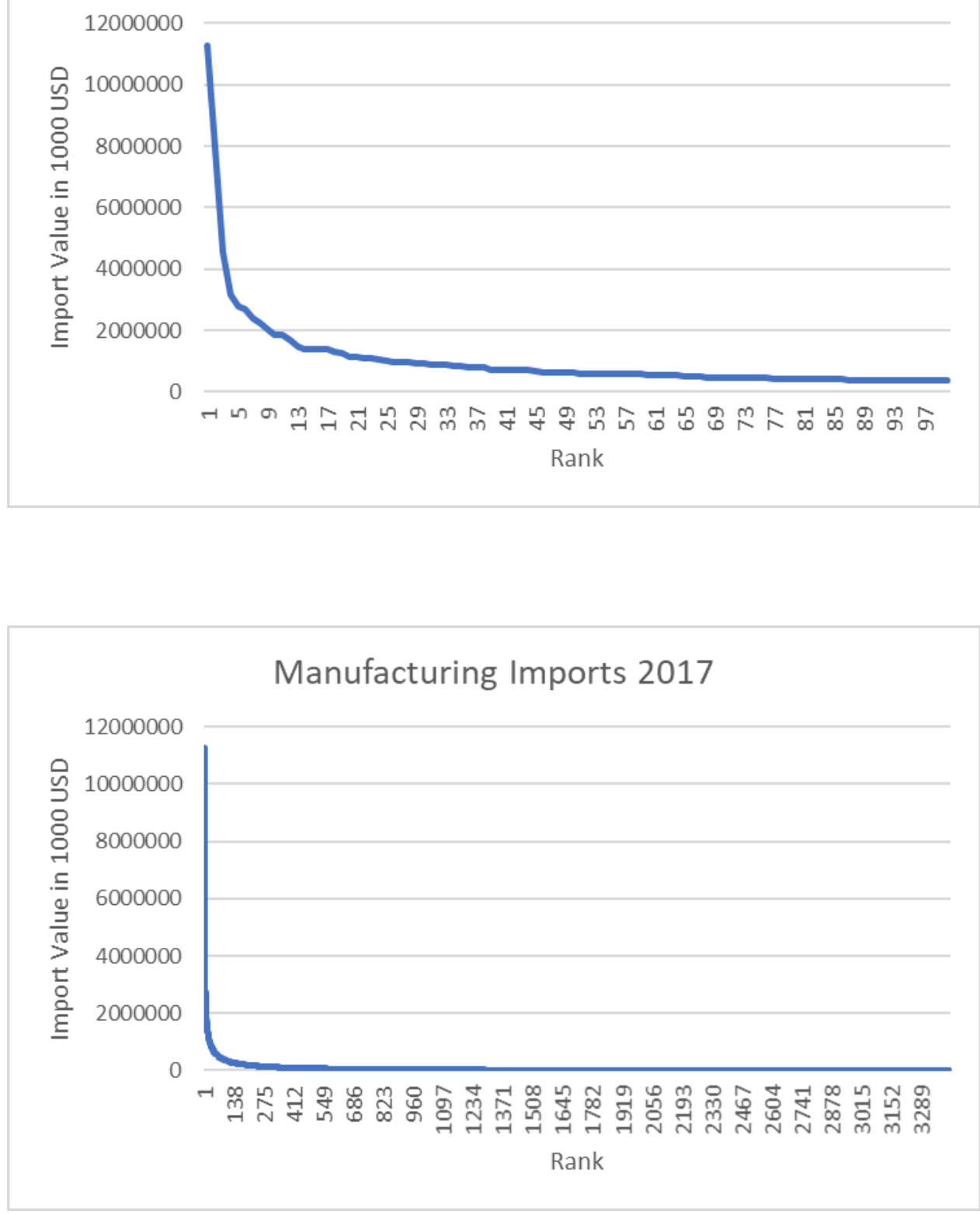

Top 100 Manufacturing Exports in 2017

12000000
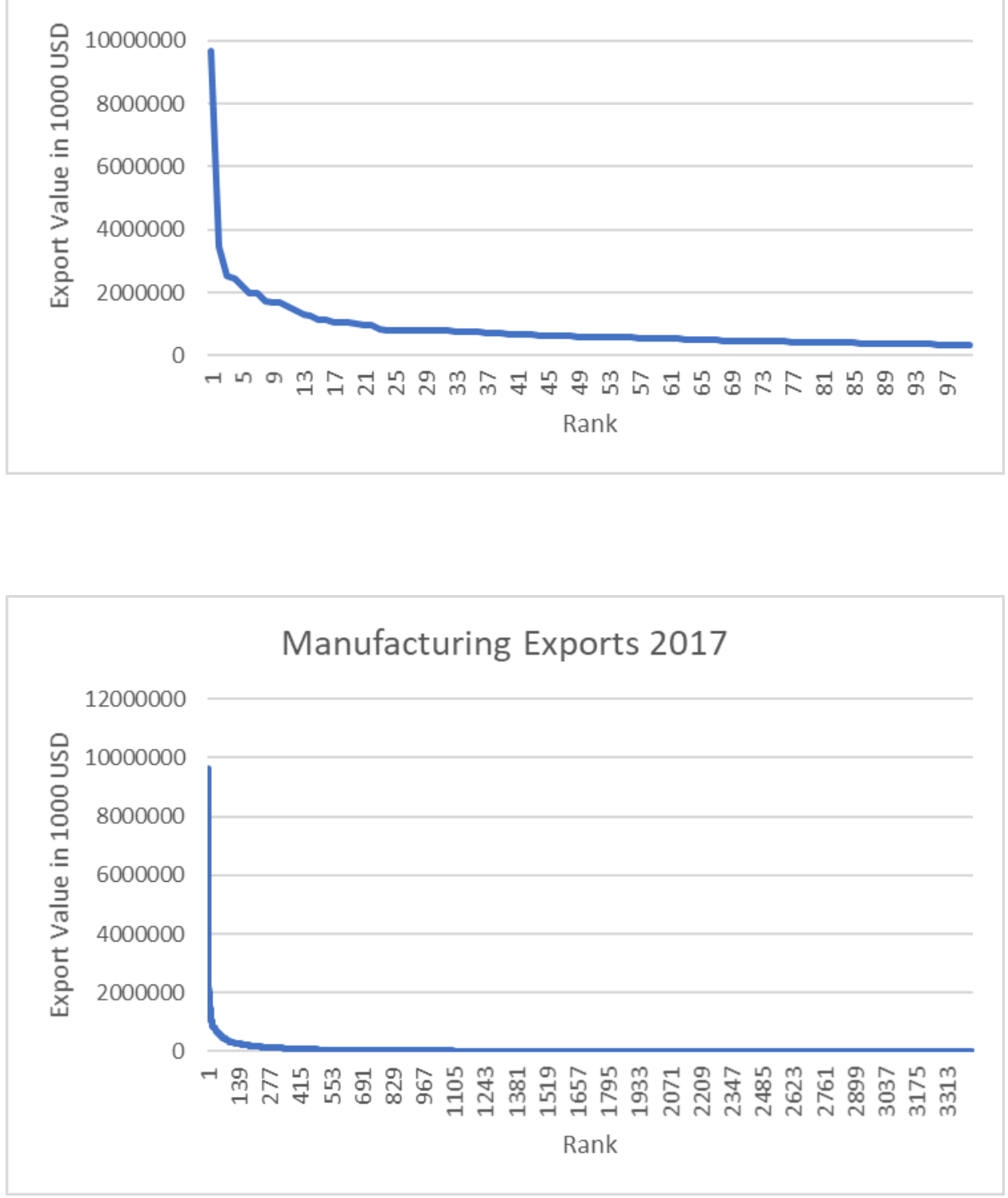


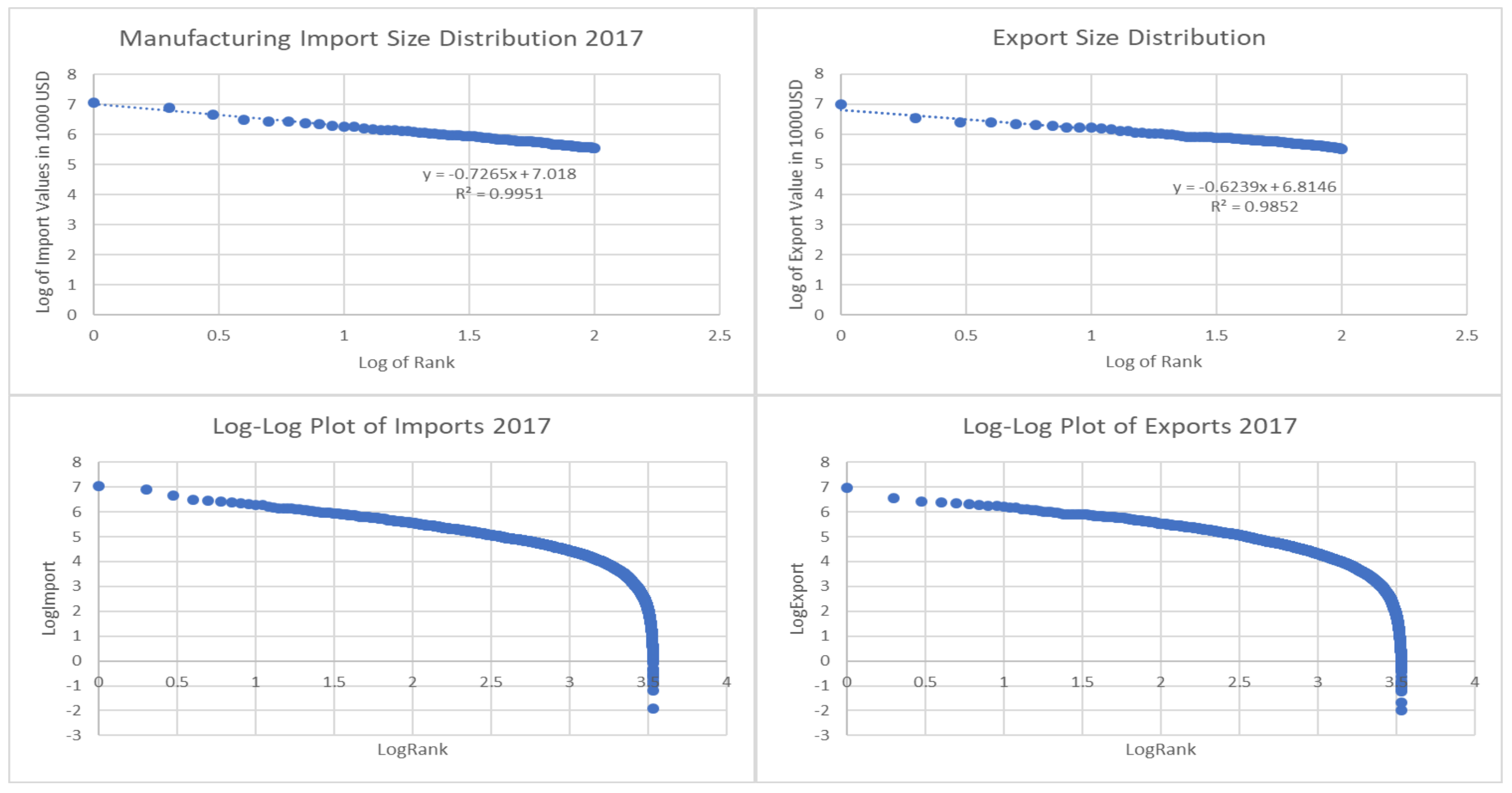




\section{Power Laws... Asymmetric Value Distribution}

- $P(x)=C x^{-\alpha}=\frac{C}{x^{\alpha}}$, for $x \geq x_{\min }$

- Normalisation $(\alpha>1)$

$1=\int_{x_{\min }}^{\infty} P(x) d x=C \int_{x_{\min }}^{\infty} \frac{d x}{x^{\alpha}}=\frac{c}{\alpha-1} x_{\min }^{-\alpha+1} \Rightarrow c=(\alpha-1) x_{\min }^{\alpha-1}$

- Power law PDF: $P(x)=\frac{\alpha-1}{x_{\min }}\left(\frac{x}{x_{\min }}\right)^{-\alpha}$

- Complementary CDF: $\bar{F}(x)=\operatorname{Pr}(X>x)=\int_{x}^{\infty} P(x) d x=\left(\frac{x}{x_{\min }}\right)^{-(\alpha-1)}$

$$
\bar{F}(x)=\bar{C} x^{-(\alpha-1)} \Rightarrow \log \bar{F}(x)=\log \bar{c}-(\alpha-1) \log x
$$




$$
\begin{aligned}
& \log \operatorname{rank}_{i}=\text { constant }-\alpha \log X_{i}+\varepsilon_{i} \\
& \log \left(\operatorname{rank}_{i}-0.5\right)=\text { constant }-\alpha \log X_{i}+\varepsilon_{i} \\
& p_{(r)}=C \frac{(n+1-r)^{b}}{r^{a}} \\
& \log \left(p_{(r)}\right)=c_{0}+c_{1} \log (r)+c_{2} \log \left(r^{\prime}\right) \\
& r^{\prime}=n+1-r, c_{1}=-a, c_{2}=b, \text { and } c_{0}=\log (C) .
\end{aligned}
$$



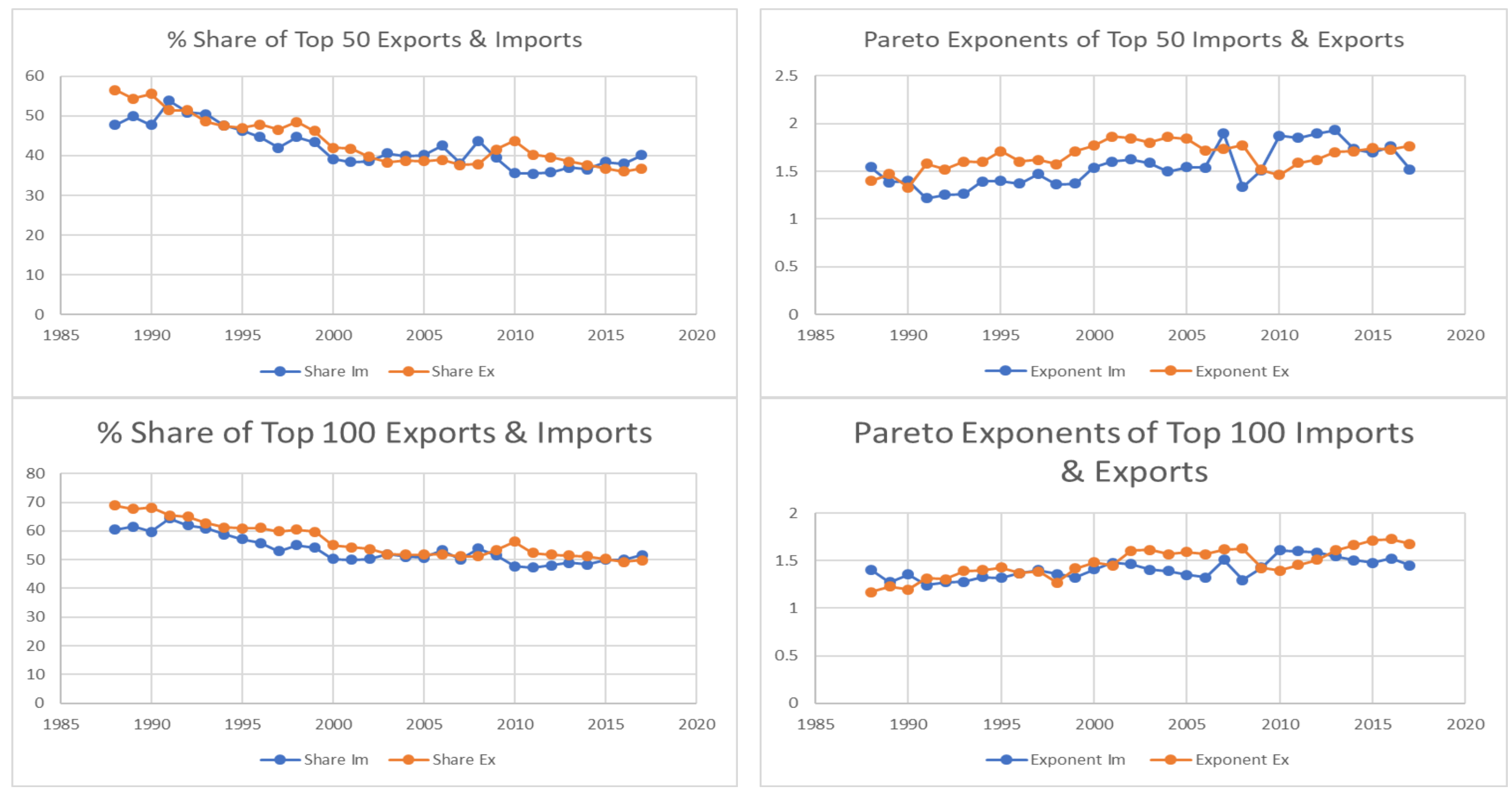
1st Exponent

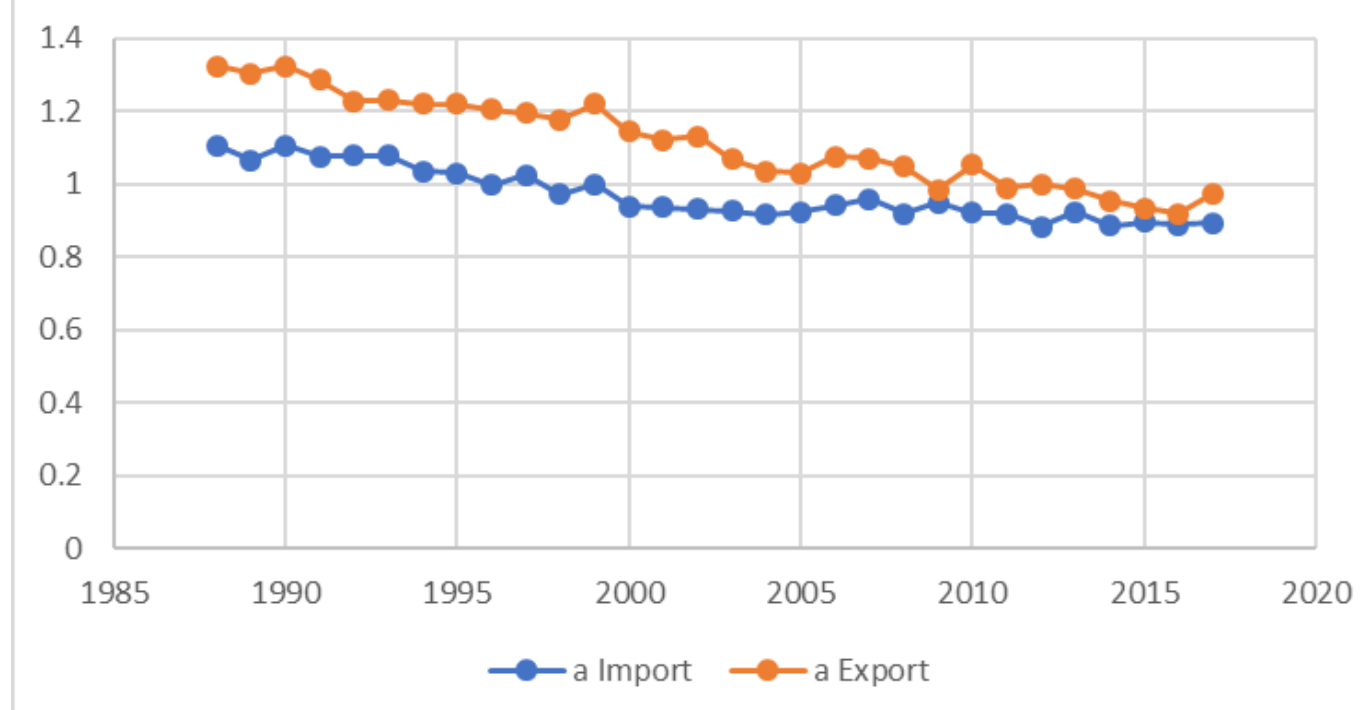

Entropy

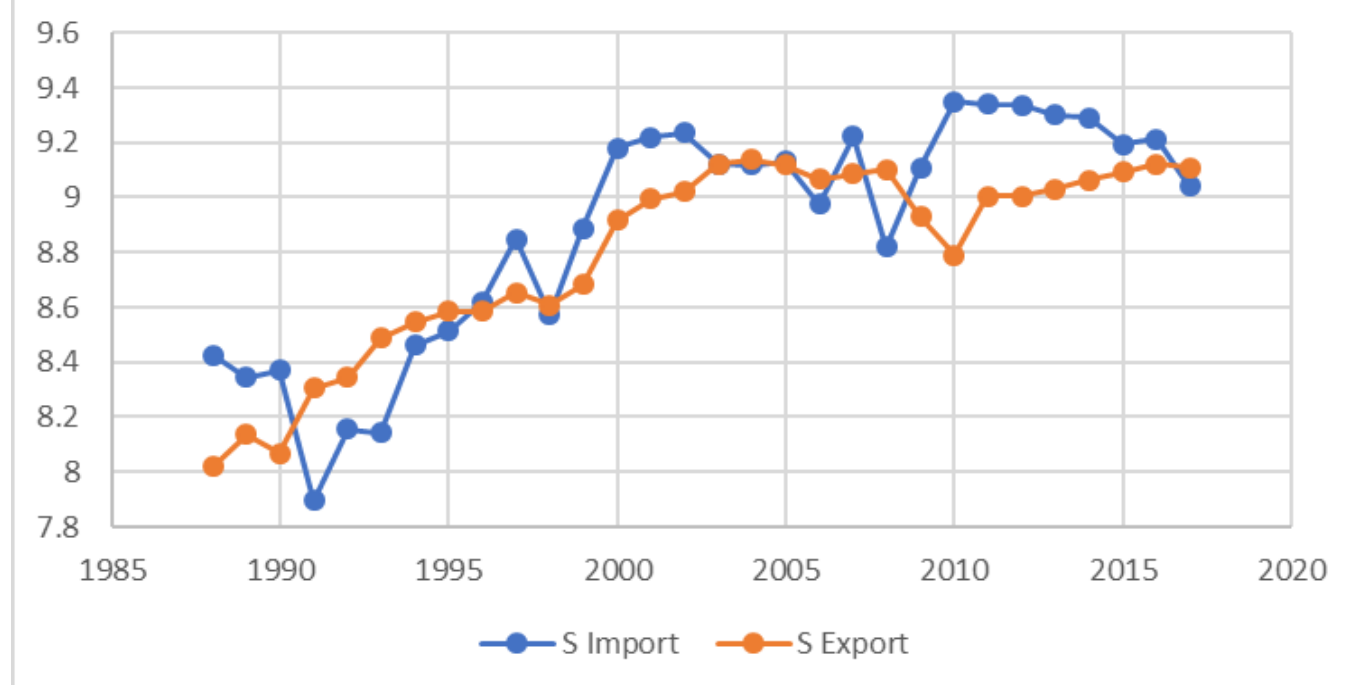

2nd Exponent

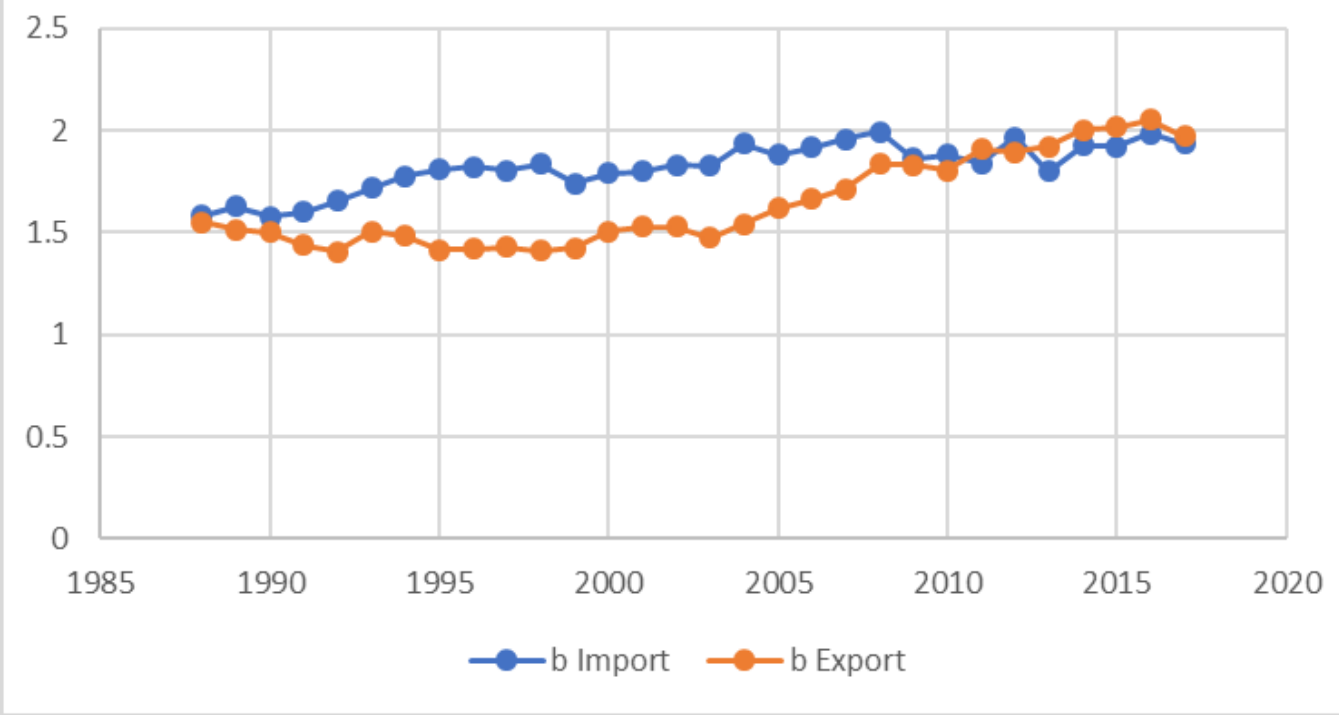

\# Products

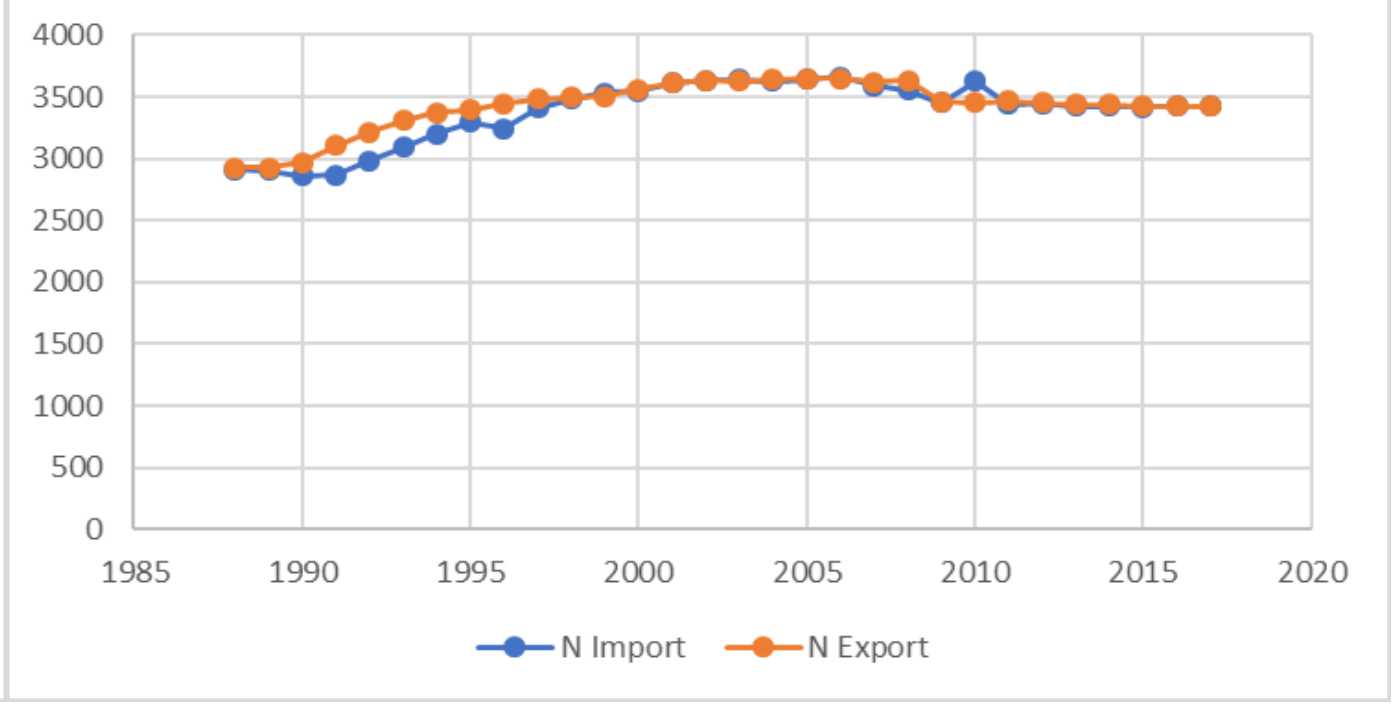


Manufacturing Imports (Stages of Processing)

\begin{tabular}{|c|c|c|}
\hline Raw Materials & $\begin{array}{c}\text { Intermediate } \\
\text { Goods }\end{array}$ & Consumer Goods \\
\hline Primary & Primary \\
\hline Resource-based & Primary \\
\hline Low-tech & Rosource-based \\
\hline Medium-tech & Low-tech \\
\hline
\end{tabular}

Import

to

Export

\section{Economy (Box of Processing)}

\begin{tabular}{|c|c|}
\hline Raw Materials & $\begin{array}{c}\text { Intermediate } \\
\text { Goods }\end{array}$ \\
\hline Primary & Grimary \\
\hline Resource-based & Resource-based \\
\hline Low-tech & Low-tech \\
\hline Medium-tech & Medium-tech \\
\hline
\end{tabular}

Consumer Goods

Primary

Resource-based

Low-tech

Medium-tech

High-tech

\section{Capital Goods}

Low-tech

Medium-tech

High-tech 